\title{
Schwannoma of stomach; a rare cause of upper GI bleeding
}

\author{
Keppetiyagama CT, Fernando A \\ Teaching Hospital, Karapitiya, Galle, Sri Lanka \\ Correspondence: Dr. Chathuranga Keppetiyagama (chathurangakap@gmail.com)
}

\section{Introduction}

Schwannoma, a benign tumor of nerve sheath origin, is commonly found in the head and neck, as well as flexor surfaces of the extremities. It can occur very rarely in the stomach with only a few cases being reported. The most common gastrointestinal site is the stomach, constituting $0.2 \%$ of all gastric neoplasms (1). Malignant transformation of a gastric schwannoma is very rare (2). They generally are asymptomatic or present with abdominal discomfort or as an epigastric mass. Bleeding may occur in the case of deep ulceration. Schwannoma is diagnosed by upper gastrointestinal endoscopy and biopsy (1). Ultrasound, CT and MRI may help in further evaluation. Surgical resection is curative if it is benign but malignant counterpart requires adjuvant treatment(1).

\section{Case Report}

A 65-year old female presented with an episode of haematemesis and vomitus contained nearly $50 \mathrm{~mL}$ of fresh blood, mixed with food. She also had recent loss of weight while her appetite was normal. There was no dysphagia but she complained of abdominal bloating and a burning epigastric pain. Bowel habits and stool colour were normal. She didn't have any medical illness and was not on NSAIDS or other drugs. There was no family history of upper gastrointestinal tumours or bleeding diathesis. She was not a smoker or consumed alcohol.

She was wasted and mild pallor was noticed. Virchow's node was not palpable. There was an epigastric mass which was firm and mobile. Liver and spleen were not palpable while the examination of cardiovascular, respiratory, and neurological systems was normal.

$\mathrm{Her} \mathrm{Hb}$ was $9 \mathrm{~g} / \mathrm{dL}$ while rest of the full blood count, renal functions and clotting screen were within normal limits. Upper gastrointestinal endoscopy revealed a large fundal ulcerated growth and multiple biopsies showed histological features of a Schwannoma with background benign gastric ulcer and active chronic gastritis. While waiting for histology, ultrasound scan was performed and it showed a mass in the stomach. Her liver was normal, ultrasonically, and no intra-abdominal lymphadenopathy was seen. Upper partial gastrectomy with oesophago-gastrostomy and feeding jejunostomy were done. Post operative period was uneventful. Histology confirmed the diagnosis of benign Schwannoma.

\section{Discussion}

Schwannomas, also known as neurinomas or neurilemmomas, are generally benign, slowgrowing neoplasms originating in any nerve that has a Schwann cell sheath. These neoplasms are rare among the spindle cell mesenchymal tumors of the gastrointestinal tract, but develop most commonly in the stomach representing $0.2 \%$ of all gastric tumors (1). Gastrointestinal schwannomas are classified as mesenchymal or neuroectodermal neoplasms. These are a heterogeneous group of tumors arising from the wall of the gastrointestinal tract that include gastrointestinal stromal tumors (GISTs), leiomyomas, leiomyosarcomas, schwannomas, neurofibromas, ganglioneuromas, paragangliomas, lipomas, granular cell tumors, and glomus tumors (2). Of these, GISTs are by far the most common in the stomach and intestines. Malignant transformation of a gastric schwannoma is very rare (2), and only once has been reported in children. Gastrointestinal schwannomas occur most commonly in the stomach (nearly $60 \%$ ), followed by the colon and rectum. Oesophageal and smallintestinal schwannomas have been rarely reported.

These tumors are usually asymptomatic, but in some 
cases, bleeding or a palpable mass can be seen. Gastrointestinal endoscopy is the principal diagnostic tool and histopathology confirms the diagnosis. Immunohistochemical assay with CD 117 \& Ki 67 help confirmation of the diagnosis further (1). Computed tomography, sonography, and magnetic resonance imaging give added information. During imaging gastric schwannomas usually appear as discrete submucosal masses that are indistinguishable from other mesenchymal tumors. As they outgrow the blood supply, these lesions may undergo central necrosis and ulceration (2).

Treatment is usually local resection without lymphadenectomy $(1,3,4)$. Local resection with $1-2$ $\mathrm{cm}$ margin is the safer option. If tumour is bigger than $5 \mathrm{~cm}$, formal gastrectomy is done (3) (open or laparoscopic hand assisted). If malignant, lymphadenectomy followed by adjuvant therapy is necessary (4).

\section{References}

1. Sir Alfred Cuscheri. Disorders of the stomach and duodenum, Sir Alfred Cuschieri, Essential Surgical Practice, $4^{\text {th }}$ edition, Geogrina Bentliff, 2002: 312-4.

2. Sarlomo-Rikala M, Miettinen M. Gastric schwannoma: a clinicopathological analysis of six cases. Histopathology, 1995; 27: 355-60.

3. Winslet MC, Stomach and duodenum, Kirk RM, General Surgical Operations, $5^{\text {th }}$ edition, Elsevier, 2007: 186-9.

4. Oliver McAnna, Myles Joice, Classic operations of upper gastrointestinal tract, Margaret Farquharson, Brendan Moran, Farquharson's textbook of General Surgery, $9^{\text {th }}$ edition, Hodder education, 2005: 273-6. 